\title{
Inequalities for Semi-Stable Fibrations on Surfaces, and Their Relation to the Coleman-Oort Conjecture
}

\author{
Chris Peters \\ To my colleague and friend Eduard Looijenga, for his 69th birthday.
}

\begin{abstract}
For semi-stable fibrations in curves over a curve Arakelov established a basic inequality which can be interpreted Hodge theoretically as an estimate for the degree of the Deligne extended Hodge bundle. When equality holds, it is well known that the period map embeds the base of the fibration as a Shimura curve in the relevant quotient of the Siegel upper half space. Going back to the situation of curve fibrations, one can ask if for those the Arakelov bound is sharp. This turns out not to be the case except if the genus of the fiber is "small". This result is due to X. Lu and K. Zuo. Cf. [L-Z]. In this note, I derive a simplified proof, using detailed surface theory. I also explain the relation with Shimura curves and the Coleman-Oort conjecture.
\end{abstract}

Keywords: Arakelov inequalities, Shimura curves, semi-stable curve fibrations over curves, Coleman-Oort conjecture.

\section{Introduction}

\section{Background}

Arakelov showed $[\mathrm{Ar}]$ that over a fixed curve of genus $\geq 2$ there are at most finitely many non-constant fibrations in curves of fixed genus for which its singular fibers appear over a fixed set in the base. To prove this, he first showed that these fibrations appear in a finite number of families (boundedness) and secondly that these are all rigid. Boundedness follows from a bound involving the relative canonical bundle. This bound is the "classical" Arakelov inequality.

Received October 29, 2015. 
The moduli space $\mathcal{M}_{g}$ of genus $g$ curves is quasi-projective and admits a natural compactification, $\overline{\mathcal{M}}_{g}$, the Deligne-Mumford compactification. See e.g. [A-C-G, Chapter X]. This compactification is constructed by adding points to the boundary which correspond to (isomorphism classes of) stable curves. These are (in general reducible) non-reduced curves having ordinary double points and each smooth rational component meets the remaining components in at least 3 points. Equivalently: the fibration first of is relatively minimal, i.e. no fiber contains a $(-1)-$ curve $^{1}$, and secondly, no fiber contains an ADE-configuration. ${ }^{2}$ So, in order to have a natural morphism of $B$ into $\overline{\mathcal{M}}_{g}$ all fibers should be stable. Clearly, this is not always the case, but there is a procedure ("semi-stable reduction") explained e.g. in [B-P-H-V, III.10], which produces a fibration over a finite (ramified) covering of $B$ whose fibers in general not yet stable but semi-stable: the fibers are reduced and have only double points and do not contain $(-1)$-curves but might contain $(-2)$-curves. The latter can be blown down in the surface which produces rational double points and the resulting family has stable fibers and hence its base maps to $\overline{\mathcal{M}}_{g}$. In view of these considerations, one may often reduce the study of curve fibrations over curves to semi-stable fibrations. I shall assume this for the remainder of this introduction.

Since the Arakelov bound is essentially Hodge theoretic in nature, it is more natural to formulate it in terms of variations of Hodge structure. The weight one case correspond to families of curves through their first cohomology groups. This link leads to an an important observation: the cohomology of a singular semi-stable fiber $C$ may have the Hodge structure of a smooth fiber; then the Jacobian $\operatorname{Jac}(C)$ is a polarized abelian variety. In our setting (of semi-stable fibrations) this happens precisely when $C$ is a tree of of smooth curves, say $C_{j}, j=1, \ldots, N$ and then $\operatorname{Jac}(C)=\prod_{j=1}^{N} \operatorname{Jac}\left(C_{j}\right)$. Such a curve as well as its Jacobian is called of compact type. This shows that it is natural to separate the critical locus $\Delta$ of $f$ in two: the set of points $\Sigma_{c}$ over which the fiber is of compact type, and the remainder $\Sigma=\Delta-\Sigma_{c}$. Whereas Arakelov's original bound is expressed in terms of $\Delta$, the Hodge theoretic bound uses $\Sigma$. It involves the relative canonical sheaf ${ }^{3} \omega_{X / B}$ and

\footnotetext{
${ }^{1}$ I.e., a smooth rational curve of self-intersection $(-1)$

${ }^{2}$ I.e. a tree of smooth rational curves of self-intersection -2 resolving a rational double point.

${ }^{3}$ See Section 1.1 where the definition and basic properties are given.
} 
can be expressed as follows. ${ }^{4}$

$$
\begin{aligned}
& \operatorname{deg}\left(f_{*} \omega_{X / B}\right) \leq \frac{1}{2} g \cdot(2 g(B)-2+|\Sigma|) \\
& \text { with } g=\text { fiber genus, } g(B)=\text { base genus. }
\end{aligned}
$$

In this guise it has been shown by Faltings. Hodge theoretic generalizations to higher weights can be found in [Pe00, Jost-Zuo]. It has become common practice to call this inequality also an "Arakelov inequality".

\section{Special curve fibrations over a curve}

An interesting special case arises when the Arakelov bound is attained, i.e. when equality holds in (1). Rather surprisingly, one has [L-Z]:

Theorem (A). For a non-isotrivial genus $g \geq 5$ fibration $f: X \rightarrow B$ of semi-stable curves the Arakelov bound (1) can never be attained.

The aim of this note is first of all to give a simplified and self contained proof of Theorem (A). By using refined Chern class inequalities for noncompact surfaces obtained in [Ko, Ch-Y] instead of those from [Miy], the proof given here avoids the elaborate covering tricks employed in [L-Z]. This not only simplifies their proof but also makes the basic idea of their proof more transparent. This is the content of Sects. 1-2.2.

The main idea of the proof is as follows. For surface fibrations in semistable curves Moriwaki obtained an inequality (3) for the so called slope. The Chern class inequality cited above is a refinement (=Theorem 1.5) of the classical Bogomolov-Miyaoka-Yau inequality ${ }^{5}$. If the Arakelov bound is attained one can rewrite this as an inequality for the slope in the reverse direction. Note that this inequality depends on the boundary of the open surface. One has to choose this boundary carefully and then the combined inequalities are seen to be only possible when the fibering curves have genus at most 4 .

At this point I should mention that ideas inspired by the cited work [L-Z, L-Z2] of X. Lu and K. Zuo have been used in the reverse direction to obtain more refined slope inequalities, a result that should be of direct interest to surface geometers. See [L-T-Y-Z, L-Z3, L-Z4, L-Z5].

\footnotetext{
${ }^{4}$ One has to suppose that $2 g(B)-2+|\Sigma|>0$.

${ }^{5}$ In what follows this will be abbreviated as $B M Y$-inequality.
} 


\section{Moduli aspects}

A further aim of this note is to elucidate the moduli aspects ${ }^{6}$ of Theorem (A), especially its relation with the so-called Coleman-Oort conjecture [Co, O] about the Torelli locus. Let me explain this very briefly. As noticed before, the Arakelov inequality (1) admits a purely Hodge theoretical proof which holds for a polarized weight one variation of Hodge structure ${ }^{7}$ over a curve, or, equivalently, for a family of principally polarized Abelian varieties of dimension $g$ over a curve.

The corresponding moduli space $\mathcal{A}_{g}$ contains many obvious subvarieties coming from other Abelian varieties with extra structure, for instance those coming from products of lower dimensional Abelian varieties. Such subvarieties belong to the class of what nowadays is called the class of special subvarieties, by definition images in $\mathcal{A}_{g}$ of Shimura varieties. See $\S 3.1$ for a brief introduction to the subject of Shimura varieties. Shimura varieties of dimension 1 are called Shimura curves. Note that contrary to some practices, I do not consider them as embedded in $\mathcal{A}_{g}$, but as coming from a Shimura datum. In Section 3.2 I explain Satake's classification of Shimura curves: the non-compact Shimura curves are precisely the modular curves parametrizing elliptic curves with a fixed level structure and the compact ones come from the quotient of the upper half plane by an arithmetic subgroup of the group of units of certain quaternion algebras.

These curves come up naturally because of the following basic result, essentially due to E. Viehweg and K. Zuo (see [Vie-Z]):

Proposition (=Prop. 3.1). If equality holds in the Hodge theoretic version (18) of the Arakelov inequality, the punctured curve $B-\Sigma$ is a rigidly embedded Shimura curve. The converse also holds.

It is well known that $\mathcal{A}_{g}$ is quasi projective and contains many special subvarieties. For instance special points are dense. The many types of special subvarieties of $\mathcal{A}_{g}$ all have been classified [Ad]. It follows from this that the rigidly embedded special curves form a relatively small list:

1) fibered self-products of the modular family of elliptic curves. The corresponding embedded curves are called of Satake type;

\footnotetext{
${ }^{6} \mathrm{~A}$ modern and self-contained reference for moduli spaces of curves is [A-C-G].

${ }^{7}$ The proof of the resulting inequality (18) is reproduced below in Section 2.1.
} 
2) fibered self-products of modular families of Abelian varieties over compact Shimura curves. The corresponding embedded curves are called of Mumford type after [Mum69].

Another interesting sublocus of $\mathcal{A}_{g}$ comes from the moduli space $\mathcal{M}_{g}$ of (smooth projective) genus $g$ curves: the Torelli theorem states that the period map, which associates to a smooth genus $g$ curve $C$ its Jacobian $\operatorname{Jac}(C)$, defines an injective morphism

$$
p: \mathcal{M}_{g} \rightarrow \mathcal{A}_{g}
$$

For $g \geq 2$ the image of $p$ is not closed in $\mathcal{A}_{g}$. As is well known [Mum75, p. 74] the limits come from curves of compact type encountered before: their (generalized) Jacobian is a product of lower dimensional Abelian varieties. This non-closedness explains why, traditionally, the Torelli locus $\mathcal{T}_{g}$ is defined to be the closure inside $\mathcal{A}_{g}$ of the image of the period map.

For $g \geq 4$ the locus $\mathcal{T}_{g}$ is not special in the above sense. Indeed, it is believed that positive dimensional special subvarieties $S \subset \mathcal{A}_{g}$ cannot generically be contained in the Torelli locus 8 unless $g$ is "small". This belief goes under the name of the Coleman-Oort conjecture, see [Co, O]. In [M-O] background for this conjecture is given as well as examples why this fails for low $g$.

An important conclusion of the previous discussion is that Shimura curves which are rigidly embedded in $\mathcal{A}_{g}$ are precisely the ones for which the corresponding Hodge bundle attains the Arakelov bound. Hence, Theorem (A) clearly is related to the Coleman-Oort conjecture. Unfortunately, $\mathcal{A}_{g}$ is not a fine moduli space and so one cannot assume that there is a (tautological) family of genus $g$ curves over an embedded special curve. This explains why further work needs to be done. In [L-Z2] the authors show that one in fact has:

Theorem (B). Suppose $g \geq 12$. Then no rigidly embedded special curve (necessarily of Satake or of Mumford type) can generically be contained in $\mathcal{T}_{g}$

In Section 3.3 I shall briefly outline how this can be shown by suitably modifying the proof of Theorem (A) as given in this note. These modifications are however not straightforward and require new ideas which I don't elaborate on.

\footnotetext{
${ }^{8} S \subset \mathcal{A}_{g}$ is generically contained in $\mathcal{T}_{g}$ if it is contained in $\mathcal{T}_{g}$ but also meets $p\left(\mathcal{M}_{g}\right)$ in a Zariski open subset of $S$.
} 
Finally, I also want to remark that similar ideas enabled X. Lu and $\mathrm{K}$. Zuo [C-L-Z2] to verify the Coleman-Oort conjecture for certain higher dimensional Shimura varieties.

Acknowledgements: I thank Kang Zuo for spotting a gap in a preliminary version of my proof of Theorem A and for his hints as how to fill it. Furthermore, I want to express thanks to the anonymous referee(s) for spotting several inaccuracies and unclear passages in this modified version.

\section{Background from surface theory}

\subsection{Proportionality deviation for semi-stable fibrations}

Let $X$ be a minimal compact complex algebraic surface and suppose that

$$
f: X \rightarrow B, \text { genus } B=b,
$$

is a fibration in genus $g \geq 2$ semi-stable curves. Denote the fibre over $s$ by $X_{s} ; \operatorname{Jac}\left(X_{s}\right)$ denotes the generalized Jacobian of $X_{s}$. As usual, put

$$
\omega_{X / B}=\mathcal{O}_{X}\left(K_{X} \otimes f^{*} K_{B}^{-1}\right), \quad \text { the relative dualizing sheaf. }
$$

Recall [B-P-H-V, Theorem III, 18.2] that a relatively minimal fibration ${ }^{9}$ in genus $\geq 2$ curves $f: X \rightarrow B$ is isotrivial ${ }^{10}$ if and only if $\operatorname{deg} f_{*} \omega_{X / S}=0$. So, if $f$ is not isotrivial, one can introduce the slope

$$
\lambda(f):=\omega_{X / B}^{2} / \operatorname{deg}\left(f_{*} \omega_{X / B}\right) .
$$

Recall also that $f$ is called a Kodaira fibration if $f$ is smooth. In that case, the period map for $f$ sends $B$ to a compact curve in the moduli space $\mathcal{M}_{g}$ of genus $g$ curves. For any Kodaira fibration the fibre genus $g$ and the base genus $g(B)$ have to be at least 2. See [B-P-H-V, V. 14].

Relations between the various numerical invariants of a surface fibration are gathered in the next Lemma.

Lemma 1.1. For a relatively minimal surface fibered in genus $g$ curves $^{11}$ $f: X \rightarrow B, g(B)=b$, one has ${ }^{12}$ :

\footnotetext{
${ }^{9}$ I.e., there are no $(-1)$-curves in the fibres.

${ }^{10}$ I.e., locally constant over the non-critical locus of $f$.

${ }^{11}$ The fibers need not be semi-stable.

${ }^{12}$ Standard notation is used here: $e$ denotes the topological Euler number and $c_{1}^{2}(X)=K_{X}^{2}$
} 
(i) $\operatorname{deg}\left(f_{*} \omega_{X / B}\right)=\frac{1}{12}\left(c_{1}^{2}(X)+e(X)\right)-(b-1)(g-1)$.

(ii) $c_{1}^{2}(X)=\omega_{X / B}^{2}+8(g-1)(b-1)$.

(iii) $e(X)=4(g-1)(b-1)+\sum_{s \in B} \delta_{s}$, where

$$
\delta_{s}:=e\left(X_{s}\right)-e\left(X_{\text {gen }}\right) \geq 0, \quad e\left(X_{\text {gen }}\right)=2(1-g) .
$$

(iv) $12 \operatorname{deg} f_{*} \omega_{X / B}=\omega_{X / B}^{2}+\sum_{s} \delta_{s}$.

(v) Suppose, moreover, that $f$ is non-isotrivial and $g \geq 2$. Then

$$
4-\frac{4}{g} \leq \lambda(f) \leq 12 .
$$

The leftmost inequality is Xiao's slope inequality. The right hand inequality becomes an equality, $\lambda(f)=12$ if and only if $f$ is a Kodaira fibration.

Proof: (i) is a direct consequence of Riemann-Roch and the Leray spectral sequence.

(ii) follows from the definition of $\omega_{X / B}$.

(iii) See [B-P-H-V, Prop. III, 11.4].

(iv) Follows directly from (i),(ii), (iii).

(v) See $[\mathrm{Xiao}]$. The remaining assertion about the upper bound of $\lambda(f)$ follows immediately from (iv).

Motivated by the BMY-inequality $c_{1}^{2}(X) \leq 3 c_{2}(X)$ (see [B-P-H-V, Theorem VII, 4.1]), one introduces the proportionality deviation

$$
\beta(X):=3 c_{2}(X)-c_{1}^{2}(X) .
$$

The BMY-inequality then becomes $\beta(X) \geq 0$ with equality if and only if $X$ is a ball quotient.

Corollary 1.2. In the situation of (2), one has

$$
\beta(X)=4(b-1)(g-1)-\omega_{X / B}^{2}+3 \sum_{s \in B} \delta_{s}
$$

The invariants $\delta_{s}$ are just the number of double points of the fiber $X_{s}$. To show this, one uses expressions for the Euler number and the arithmetic genus of a semi-stable curve: 
Lemma 1.3. Let $C$ be a semi-stable curve on a compact complex surface $X$ and let the irreducible components be $C_{\alpha}, \alpha=1, \ldots, N$; the genus of the normalization of $C_{\alpha}$ is denoted $g_{\alpha}$ and $\delta_{C}$ is the number of double points of $C$. Then one has:

$$
\begin{aligned}
e(C) & =\sum_{\alpha}\left(2-2 g_{\alpha}\right)-\delta_{C} . \\
2 p_{a}(C)-2 & =K_{X} \cdot C+C^{2}=\sum_{\alpha}\left(2 g_{\alpha}-2\right)+2 \delta_{C} .
\end{aligned}
$$

Proof: The first formula follows from the additive property of the Euler number; the second can be seen as follows. For an irreducible component $C_{\alpha}$ of $X_{s}$ one has $p_{a}\left(C_{\alpha}\right)=g_{\alpha}+\delta_{\alpha}$ with $\delta_{\alpha}$ the number of double points of $C_{\alpha}$; the number $\sum_{\alpha<\beta} C_{\alpha} C_{\beta}$ is the number $\delta_{s}^{\prime}$ of those double points of $X_{s}$ that are intersections of two components. Hence

$$
\begin{aligned}
K_{X} \cdot C+C^{2} & =\sum_{\alpha}\left(K_{X} \cdot C_{\alpha}+C_{\alpha}^{2}\right)+2 \sum_{\alpha<\beta} C_{\alpha} \cdot C_{\beta} \\
& =\sum_{\alpha}\left(2 p_{a}\left(C_{\alpha}\right)-2\right)+2 \sum_{\alpha<\beta} C_{\alpha} \cdot C_{\beta} \\
& =\sum_{\alpha}\left(2 g_{\alpha}-2\right)+2(\underbrace{\sum \delta_{\alpha}+\delta_{s}^{\prime}}_{\delta_{C}}) .
\end{aligned}
$$

Corollary 1.4. Suppose $f: X \rightarrow B$ is a semi-stable genus $g$ fibration. Then

$$
\delta_{s}=\delta_{X_{s}}=\#\left(\text { double points of } X_{s}\right)
$$

Proof: The previous lemma gives

$$
e\left(X_{s}\right)=\sum\left(2-2 g_{\alpha}\right)-\delta_{X_{s}}
$$

On the other hand, the arithmetic genus $p_{a}\left(X_{s}\right)$ is independent of $s \in B$ since by the adjunction formula $2 p_{a}\left(X_{s}\right)-2=K_{X} \cdot X_{s}+X_{s}^{2}=K_{X} \cdot X_{s}$ which does not depend on $s$ since all fibers are numerically equivalent. So, applying the previous lemma once again, one finds

$$
\begin{aligned}
e\left(X_{\text {gen }}\right)=2-2 g & =-\left(K_{X} \cdot X_{s}+X_{s}^{2}\right) \\
& =\sum\left(2-2 g_{\alpha}\right)-2 \delta_{X_{s}} .
\end{aligned}
$$

Comparing this with (7) and using (4), shows that

$$
\delta_{s}:=e\left(X_{s}\right)-e\left(X_{\text {gen }}\right)=\delta_{X_{s}} .
$$




\subsection{A refined BMY-inequality}

There is a refinement of the BMY-inequality due to R. Kobayashi [Ko], and Cheng-Yau [Ch-Y]. An algebraic proof has been given by Miyaoka [Miy], but that proof gives no information in case of equality, information which is crucial for the proof I give of Theorem (A).

To state it, some preparations are needed. First, recall that a divisor $D$ on a surface is big if for $m \gg 0$ the linear system $|m D|$ maps $X$ birationally onto its image. This is equivalent to $\kappa(D)=2$, where $\kappa$ denotes the Kodairadimension. If some multiple of $D$ is effective, $D$ is big precisely if $D^{2}>0$ [B-P-H-V, IV Prop. 7.4]. The divisor $D$ is nef, if $D \cdot C \geq 0$ for all curves $C$.

A surface $X$ by definition is of general type if the canonical divisor $K_{X}$ is big, or, equivalently, if $\kappa(X)=\kappa\left(K_{X}\right)=2$. A suitable multiple $\left|m K_{X}\right|$ thus maps $X$ birationally onto its image. In this situation $K_{X}$ is nef precisely when $X$ is minimal. Even then, the bundle $K_{X}$ need not be ample: $\left|m K_{X}\right|$ contracts all ADE-curve configurations. Such a configuration of curves contracts to a rational double point under $\left|m K_{X}\right|, m \gg 0$. Any such rational double point $p$ is a quotient singularity: its germ is of the form $U_{p} / G_{p}$ where $U_{p}$ is smooth and $G_{p}$ a finite group.

The refinement gives an inequality, valid for the so-called logarithmic Chern classes for a Zariski-open surface $X-C$ where $C \subset X$ is a normal crossing curve. These are defined as follows:

$$
\begin{aligned}
c_{2}(X, C) & :=c_{2}(X)-e(C) \\
c_{1}^{2}(X, C) & :=c_{1}^{2}\left(\Omega_{X}^{1}(\log C)\right)=c_{1}^{2}(X)+2 K_{X} \cdot C+C^{2} .
\end{aligned}
$$

Introduce the logarithmic proportionality deviation

$$
\begin{aligned}
\beta(X, C): & =3 c_{2}(X, C)-c_{1}^{2}(X, C) \\
& =\underbrace{3 c_{2}(X)-c_{1}^{2}(X)}_{\beta(X)}-\underbrace{\left(3 e(C)+2 K_{X} \cdot C+C^{2}\right)}_{\beta(C)} .
\end{aligned}
$$

For every A-D-E configuration $R_{p}$, contracting to a singular point $p$, one introduces also the following punctual proportionality deviation:

$$
\beta(p)=3\left(e\left(R_{p}\right)-\frac{1}{\left|G_{p}\right|}\right)>0 .
$$

Now I can state the promised strengthening of the BMY-inequality which results from Yau's techniques: 
Theorem 1.5 ([Ko, Thm 2], [Miy, Ch-Y]). Let $X$ be a compact complex surface, $C \subset X$ a normal crossing divisor such that $K_{X}+C$ is nef and big. Let $R_{p_{j}}, j=1, \ldots, k$ be the $A-D-E$ configurations in $X-C$ and let $X^{\prime}$ be the normal surface obtained after contracting the $R_{p_{j}}$ to a singularity $p_{j}$. Then

$$
\beta(X, C)-\sum_{j} \beta\left(p_{j}\right) \geq 0
$$

and equality holds if and only if $X^{\prime}-C=\Gamma \backslash B^{2}$, the quotient of the 2-ball $B^{2}$ by a discrete subgroup $\Gamma \subset \operatorname{PSU}(2,1)$ acting freely except over the singularities of $X^{\prime}$ where $\Gamma$ acts with isolated fixed points.

Remark 1.6. If $K_{X}$ itself is nef, the result applies to the following two sorts of curves $C$ that are relevant here:

1) any semi-stable curve on $X$ of arithmetic genus $\geq 2$;

2) an elliptic curve with negative self intersection.

3) disjoint unions of curves of the above sort.

To see that the condition of the theorem holds for such curves, argue as follows. Let $C=F+R$ with $F$ a disjoint union of curves as in 1 , and $R$ a disjoint union of curves as in 2. If $F \neq \varnothing$, then by nefness of $K_{X},\left(K_{X}+\right.$ $C)^{2} \geq\left(K_{X}+C\right) C=2 p_{a}(C)-2>0$. Otherwise, $\left(K_{X}+R\right)^{2}=K_{X}^{2}-R^{2}>$ 0 .

Next, again using nefness of $K_{X}$, one has $\left(K_{X}+C\right) D \geq 0$ for all irreducible curves $D$ that are not components of $C$ and thirdly, for all components $C_{i}$ of $C$ with $p_{a}\left(C_{i}\right)>0$, one has $\left(K_{X}+C\right) \cdot C_{i} \geq K_{X} C_{i}+C_{i}^{2}=$ $2 p_{a}\left(C_{i}\right)-2 \geq 0$. Finally, a smooth rational component $C_{i}$ of $C$ must meet the union $D_{i}$ of the other components in at least 2 points (this is a consequence of the definition of semi-stablity) so that

$$
\left(K_{X}+C\right) \cdot C_{i}=K_{X} \cdot C_{i}+C_{i}^{2}+D_{i} \cdot C_{i} \geq-2+2 \geq 0 .
$$

So $K_{X}+C$ is big indeed. See also [Sak, Theorem 7.6.].

\subsection{Properties of excess invariants for curves}

Lemma 1.7. 1) The excess invariant $\beta(C)$ is additive: for disjoint curves $C$ and $D$ one has $\beta(C \coprod D)=\beta(C)+\beta(D)$.

2) Let $C=\sum C_{\alpha}$ be a semi-stable curve with irreducible components $C_{\alpha}$ and 
total number $\delta_{C}$ of double points. Then, recalling that $g_{\alpha}$ is the genus of the normalization of $C_{\alpha}$, one has:

$$
\beta(C)=\sum_{\alpha}\left(2-2 g_{\alpha}\right)+\delta_{C}-C^{2} .
$$

Proof: 1) is an easy verification and 2) follows immediately from Lemma 1.3 since

$$
\begin{aligned}
\beta(C) & =3 e(C)+2 K_{X} C+C^{2} \\
& =3 e(C)+2\left(K_{X} C+C^{2}\right)-C^{2} \\
& =3 e(C)+4 \sum_{\alpha}\left(g_{\alpha}-1\right)+4 \delta_{C}-C^{2} \\
& =6 \sum_{\alpha}\left(1-g_{\alpha}\right)-3 \delta_{C}+4 \sum_{\alpha}\left(g_{\alpha}-1\right)+4 \delta_{C}-C^{2} .
\end{aligned}
$$

Examples 1.8. 1) Let $C=X_{s}$ a possibly singular fibre of a semi-stable fibration in curves of genus $g$. Since $X_{s}^{2}=0$, using (8) in the formula for $\delta_{s}=\delta_{X_{s}}$ gives:

$$
\beta\left(X_{s}\right)=3 \delta_{s}+2(1-g)
$$

This can indeed be positive and in that case gives an amelioration of the BMY-inequality.

(2) Consider a fibre $X_{s}$ as in (2) of the form $X_{s}=E_{s}+F_{s}+R$ with $p_{a}\left(E_{s}\right)=$ $1, F_{s}$ a tree of smooth rational curves meeting $E_{s}$ and $R$ transversally in one point and $R$, the union of the remaining components. Note that if $X_{s}$ is of compact type, $E_{s}$ is necessarily a smooth elliptic curve and one can take for $F_{s}$ the tree of all rational curves (necessarily smooth) connecting $E_{s}$ with other curves of genus $>0$. While the curve $E_{s}$ is called an elliptic tail, the divisor $F_{s}$ is the corresponding connecting rational tail of $X_{s}$. By Remark 1.6 the semi-stable divisor $E_{s}$ can play the role of $C$ in Theorem 1.5, but this is not the case for $E_{s}+F_{s}$ since $K_{X}+E_{s}+F_{s}$ not nef: the last component of $F_{s}$ has negative intersection with $K_{X}+E_{s}+F_{s}$. Fortunately, the divisor $F_{s}$ forms a chain of $(-2)$-curves and hence can be contracted to a rational double point, say $f_{s}$, of type $A_{\ell_{s}}$. Make now note of the following inequalities

$$
\begin{cases}\beta\left(E_{s}\right)=-E_{s}^{2} \geq 1=3 \ell_{s}+1 & \text { if } F_{s}=\varnothing \\ \beta\left(f_{s}\right)=3\left(\ell_{s}+1-\frac{1}{\ell_{s}+1}\right) \geq 3 \ell_{s}+1 & \text { otherwise }\end{cases}
$$

where Lemma 1.7 and (9) have been used. 
The preceding remarks have the following useful consequence. Recall that a fiber $X_{s}$ is a singular curve of compact type if and only if its dual graph is a tree and all components of $X_{s}$ are smooth.

Corollary 1.9 (of Theorem 1.5). Let $X$ be a compact complex surface,

$$
f: X \rightarrow B
$$

a semi-stable fibration of genus $g \geq 2$ curves. Let $\Sigma \subset B$ be the set of critical points of $f$ over which the fiber is not of compact type. Let $R$ be the disjoint union of the elliptic tails in fibers of compact type that have no connecting rational tails and let $f_{j}, j \in J$ be the rational double points obtained by contracting the connecting rational tails. Setting $C=R+\coprod_{s \in \Sigma} X_{s}$, assume that $K_{X}+C$ is nef and big. Then one has

$$
2(g-1) \operatorname{deg} \Omega_{B}^{1}(\log (\Sigma))-\omega_{X / B}^{2}+3 \sum_{s \in \Sigma_{c}} \delta_{s}-\beta(R)-\sum_{j \in J} \beta\left(f_{j}\right) \geq 0
$$

Proof: Let me calculate $\beta(X, C)$. Additivity of $\beta$ and (11) give

$$
\beta(C)=3 \sum_{s \in \Sigma} \delta_{s}+\beta(R)+2(1-g) \cdot|\Sigma| .
$$

On the other hand, by Cor. 1.2 , setting $b=\operatorname{genus}(B)$, one has

$$
\begin{aligned}
\beta(X) & =4(b-1)(g-1)-\omega_{X / B}^{2}+3 \sum_{s \in B} \delta_{s} \\
& =2(g-1) \operatorname{deg} \Omega_{B}^{1}(\log (\Sigma))-\omega_{X / B}^{2}+2(1-g) \cdot|\Sigma|+3 \sum_{s \in B} \delta_{s}
\end{aligned}
$$

and so

$$
\begin{aligned}
\beta(X, C) & =\beta(X)-\beta(C) \\
& =2(g-1) \operatorname{deg} \Omega_{B}^{1}(\log (\Sigma))-\omega_{X / B}^{2}+3 \sum_{s \in B} \delta_{s}-3 \sum_{s \in \Sigma} \delta_{s}-\beta(R) \\
& =2(g-1) \operatorname{deg} \Omega_{B}^{1}(\log (\Sigma))-\omega_{X / B}^{2}+3 \sum_{s \in \Sigma_{c}} \delta_{s}-\beta(R) .
\end{aligned}
$$

So the desired inequality follows from Theorem 1.5. 
Remark 1.10. The inequality (13) will be used in the following form:

$$
\left.\begin{array}{c}
4\left(1-\frac{1}{g}\right)+\frac{1}{d(f)} \cdot\left(\sum_{s \in \Sigma_{c}} 3 \delta_{s}-\beta(R)-\sum_{j \in J} \beta\left(f_{j}\right)\right) \geq \frac{\omega_{X / B}^{2}}{d(f)} \\
d(f):=\frac{g}{2} \operatorname{deg} \Omega_{B}^{1}(\log (\Sigma)) .
\end{array}\right\}
$$

\subsection{On Moriwaki's slope inequality}

Xiao's slope inequality (5) admits a refined version which is due to Moriwaki. It is a consequence of certain enumerative properties of cycles on the compactified moduli space $\overline{\mathcal{M}}_{g}$. To explain it, let me introduce some more notation. The moduli space $\overline{\mathcal{M}}_{g}$ has as boundary the irreducible divisors $\Delta_{j}$, $j=0, \ldots,\left[\frac{g}{2}\right]$ where the stable genus $g$ curve $C$ belongs to $\Delta_{0}$ if $C$ is irreducible and to $\Delta_{j}, j>0$, if it is of the form $C=C_{1}+C_{2}$ where $p_{a}\left(C_{1}\right)=j$, $p_{a}\left(C_{2}\right)=g-j$. The double point $C_{1} \cap C_{2}$ is then called a double point of type $j$. The double points of an irreducible curve are called of type 0 . This terminology extends to the semi-stable situation: if there is a tree $R$ of rational curves connecting $C_{1}$ and $C_{2}$, all of the double points on $R$ are of type $j$.

If $p: B \rightarrow \overline{\mathcal{M}}_{g}$ is the period map for $f$, the curve $p(B)$ meets the divisor $\Delta_{j}$ exactly in the points $s \in B$ over which there is a singular fibre with a double point of type $j$. Let $\delta_{j}(f)$ be the total number of such points. Moriwaki's inequality [Mor, Theorem D] reads:

$$
(8 g+4) \operatorname{deg} f_{*} \omega_{X / B} \geq g \cdot \delta_{0}(f)+4 \sum_{j=1}^{\left[\frac{g}{2}\right]} j(g-j) \cdot \delta_{j}(f)
$$

To see that this is a refinement of the slope inequality (5), observe that by Lemma $1.4 \sum_{s \in B} \delta_{s}$ is the total number of double points $=\sum_{j=0}^{\left[\frac{g}{2}\right]} \delta_{j}(f)$ and so the right hand side of (15) reads

$$
\begin{aligned}
g\left(\sum_{s \in B} \delta_{s}\right) & +\sum_{j=1}^{\left[\frac{g}{2}\right]}(4 j(g-j)-g) \cdot \delta_{j}(f) \\
& =g\left(12 \operatorname{deg} f_{*} \omega_{X / B}-\omega_{X / B}^{2}\right)+\sum_{j=1}^{\left[\frac{g}{2}\right]}(4 j(g-j)-g) \cdot \delta_{j}(f) .
\end{aligned}
$$


Note that the second line uses Lemma 1.1.(iv). Dividing the inequality (15) by $g \cdot \operatorname{deg} f_{*} \omega_{X / B}$ then indeed leads to the sharpening of (5):

$$
\lambda(f) \geq 4-\frac{4}{g}+\frac{1}{\operatorname{deg} f_{*} \omega_{X / B}}\left[\sum_{j=1}^{\left[\frac{g}{2}\right]}\left(4 \frac{j(g-j)}{g}-1\right) \cdot \delta_{j}(f)\right]
$$

\section{Background from Hodge theory}

\subsection{Higgs bundles and the Arakelov inequality}

For background on Hodge theory used in this section see for example [Ca-MS-P, Chapter 13.1,13.2] and [PeSt, Chapter 11].

The central concept in this section is that of a Higgs bundle:

Definition 2.1. A (logarithmic) Higgs bundle on a curve $B$ with poles in a finite set $\Sigma \subset B$ is a vector bundle $\mathcal{H}$ on $B$ with a sheaf morphism $\sigma: \mathcal{H} \rightarrow \mathcal{H} \otimes \Omega_{B}^{1}(\log \Sigma)$, the Higgs field. ${ }^{13}$ A graded Higgs bundle $\mathcal{H}=\oplus \mathcal{H}^{j}$ is a Higgs bundle verifying verify $\sigma \mid \mathcal{H}^{j} \rightarrow \mathcal{H}^{j-1} \otimes \Omega_{B}^{1}(\log \Sigma)$.

A variation of Hodge structure naturally gives a graded Higgs bundle with Higgs field induced by the Gauss-Manin connection. Let me explain this in the current situation. So assume that one has a polarized variation of Hodge structure of weight 1 over $B_{0}=B-\Sigma$ with underlying local system $\mathbf{H}$. The local monodromy of $\mathbf{H}$ around points of $\Sigma$ is assumed to be unipotent. ${ }^{14}$ The vector bundle $\mathbf{H} \otimes_{\mathbf{C}} \mathcal{O}_{B^{0}}$ together with the Gauss-Manin connection then turns out to have a canonical extension as a vector bundle $\mathcal{H}$ on all of $B$ equipped with a connection with logarithmic poles in points of $\Sigma$, say

$$
\nabla: \mathcal{H} \rightarrow \mathcal{H} \otimes_{\mathbf{C}} \Omega_{S}^{1}(\log \Sigma) .
$$

The Hodge bundle $\mathcal{F}^{1} \subset \mathbf{H} \otimes_{\mathbf{C}} \mathcal{O}_{B^{0}}$ admits a unique extension

$$
\mathcal{H}^{1,0} \subset \mathcal{H}
$$

and hence a two-step filtration $\mathcal{H}^{1,0} \subset \mathcal{H}$. On the associated graded bundle $\mathcal{H}_{\mathrm{Hgs}}=\mathcal{H}^{1,0} \oplus \mathcal{H}^{0,1}$ the logarithmic connection $\nabla$ defines the structure of a

\footnotetext{
${ }^{13}$ Higgs fields $\sigma$ over a higher dimensional base also have to satisfy $\sigma \wedge \sigma=0$.

${ }^{14} \mathrm{In}$ the geometric situation this is always the case since the degenerating fibers are semi-stable.
} 
Higgs bundle with (logarithmic) Higgs field

$$
\sigma: \mathcal{H}^{1,0} \rightarrow \mathcal{H}^{0,1} \otimes \Omega_{B}^{1}(\log \Sigma) .
$$

A graded Higgs subbundle of $\mathcal{H}_{\mathrm{Hgs}}$ consists of a graded holomorphic subbundle preserved by $\sigma$. If such a subbundle comes from a local subsystem of C-vector spaces, and the Higgs field vanishes on it, the polarization makes the local system into a unitary local system. ${ }^{15}$ On the other side of the spectrum we may have Higgs subbundles for which the Higgs field is an isomorphism.

A central property is the semi-stability of Higgs bundles:

Proposition 2.2. The first Chern form of a graded Higgs subbundle $\mathcal{G}$ of a Higgs bundle $\mathcal{H}$ coming from a variation of Hodge structure on $B-\Sigma$ (with respect to the Hodge metric) is negative semidefinite, and it is 0 everywhere if and only if $\mathcal{G}^{\perp}$ is a (holomorphic) graded Higgs subbundle as well. In this case the variation splits as an orthogonal direct sum of Higgs bundles $\mathcal{H}=\mathcal{G} \oplus \mathcal{G}^{\perp}$.

For an elementary proof see [Ca-MS-P, Chapter 13.1]. For the weight one case, the above corollary applies in particular to the maximal unitary subsystem $\mathcal{H}_{\mathrm{un}}$ on which $\sigma=0$. Its Chern form is identically zero and hence by Prop. 2.2 there is an orthogonal sum decomposition

$$
\left(\mathcal{H}_{\mathrm{Hgs}}, \sigma\right)=\left(\mathcal{H}_{\max }, \sigma\right) \oplus\left(\mathcal{H}_{\mathrm{un}}, 0\right)
$$

where the first Higgs bundle has injective Higgs field.

Semi-stability implies the following Arakelov type inequality:

Theorem 2.3 ([Fal] $)$. Let $\left(\mathcal{H}_{\mathrm{Hgs}}, \sigma\right)$ be a graded Higgs bundle over the curve $B$ with poles in $\Sigma$ induced by a polarized weight one variation of Hodge structure as sketched above. Assume that $2 g(B)-2+|\Sigma|>0$. The following inequalities for the Higgs component $\mathcal{H}^{1,0}$ hold:

$$
0 \leq \operatorname{deg} \mathcal{H}^{1,0} \leq \frac{1}{2} \cdot \operatorname{rank} \mathcal{H}^{1,0} \cdot \operatorname{deg} \Omega_{X / B}^{1}(\log \Sigma)
$$

Moreover, if the Arakelov bound for $\mathcal{H}^{1,0}$ is attained, then $\mathcal{H}_{\mathrm{Hgs}}$ has no unitary subsystem, i.e. the splitting (17) just reads $\mathcal{H}_{\mathrm{Hgs}}=\mathcal{H}_{\max }$, and, moreover, the Higgs field is an isomorphism.

\footnotetext{
${ }^{15}$ If this system would be defined over $\mathbf{Q}$ its monodromy would be finite and the Higgs subbundle corresponds to an isotrivial subsystem.
} 
Conversely, if the Higgs field for $\mathcal{H}_{\mathrm{Hgs}}$ is an isomorphism, the Arakelov bound for $\mathcal{H}^{1,0}$ is attained.

Proof: The first inequality is easy: $\left(\mathcal{H}^{1,0}, 0\right)$ is a quotient Higgs bundle of $\left(\mathcal{H}_{\mathrm{Hgs}}, \sigma\right)$ and hence, by semi-stability (Theorem 2.2 ) has degree $\geq 0$.

For the second inequality, note that the degree of the components of the Higgs bundle $\mathcal{H}_{\mathrm{Hgs}}$ and $\mathcal{H}_{\max }$ are the same while the Arakelov inequality also holds for $\mathcal{H}_{\max }$. So, if the Arakelov inequality is attained for $\mathcal{H}_{\mathrm{Hgs}}$, one must have $\mathcal{H}_{\mathrm{Hgs}}=\mathcal{H}_{\max }$. I shall assume this from here on. Consider the Higgs bundle saturation $\mathcal{G}=\mathcal{H}^{1,0} \oplus \mathcal{G}^{0,1}$ for $\mathcal{H}^{1,0}$, i.e. we consider

$$
\sigma: \mathcal{H}^{1,0} \rightarrow \mathcal{G}^{0,1} \otimes \Omega_{B}^{1}(\log \Sigma)
$$

where by definition of the saturation the right hand side is the image of the Higgs field. The right side has degree $\left(\operatorname{rank} \mathcal{G}^{0,1}\right) \cdot \operatorname{deg} \Omega_{X / B}^{1}(\log \Sigma)$ and since the pair $(\operatorname{ker} \sigma, 0)$ is obviously a Higgs subbundle of $\left(\mathcal{H}_{\mathrm{Hgs}}, \sigma\right)$, by Cor. 2.2, its degree is non-positive which implies

$$
\operatorname{deg} \mathcal{H}^{1,0} \leq \operatorname{deg} \mathcal{G}^{0,1}+\left(\operatorname{rank} \mathcal{G}^{0,1}\right) \cdot \operatorname{deg} \Omega_{X / B}^{1}(\log \Sigma)
$$

On the other hand, by stability $\operatorname{deg} \mathcal{G}=\operatorname{deg} \mathcal{H}^{1,0}+\operatorname{deg} \mathcal{G}^{0,1} \leq 0$ and hence, adding these two ineqalities, the second inequality follows. Moreover, if equality holds, $\operatorname{deg}(\mathcal{G})=0$ and $\mathcal{G}=\mathcal{H}_{\mathrm{Hgs}}$ so that $\sigma$ is surjective. Since $\mathcal{H}_{\text {un }}=$ 0 , the Higgs field has no kernel and hence $\sigma$ is an isomorphism. The proof of the converse statement is left to the reader.

Corollary 2.4. Let $f: X \rightarrow B$ be a family of semi-stable curves of genus $g$ over a curve $B$ with $\Sigma \subset B$ the subset of those critical points $s \in B$ of $f$ for which $\operatorname{Jac}\left(X_{s}\right)$ is not compact. For the associated logarithmic Higgs bundle $\left(\mathcal{H}_{f}, \sigma_{f}\right)$ let $\mathcal{H}_{f}=\mathcal{H}_{f, \max } \oplus \mathcal{H}_{f, \text { un }}$ be the splitting (17). As before, assume that $2 g(B)-2+|\Sigma|>0$. With $g_{0}$ the rank of the bundle $\mathcal{H}_{f, \mathrm{un}}^{1,0}$, the Arakelov inequality refines to

$$
\operatorname{deg} \mathcal{H}_{f}^{1,0}=\operatorname{deg} \mathcal{H}_{f, \max }^{1,0} \leq \frac{1}{2} \cdot\left(g-g_{0}\right) \cdot \operatorname{deg} \Omega_{X / B}^{1}(\log \Sigma) .
$$

The Arakelov bound for the full Hodge bundle $\mathcal{H}_{f}^{1,0}$ is attained, i.e.,

$$
\operatorname{deg} f_{*} \omega_{X / B}=\frac{1}{2} g \cdot \operatorname{deg} \Omega_{B}^{1}(\log \Sigma)
$$

if and only if $\mathcal{H}_{f}=\mathcal{H}_{f, \max }$ and the Higgs field $\sigma_{f}$ is an isomorphism. 
Remark 2.5. The proof shows that the Arakelov bound for $\mathcal{H}_{f, \max }^{1,0}$ is attained if and only if its own Higgs field is an isomorphism.

Below I also need a rigidity result for Higgs bundles. Recall that a deformation of a family $f: Y \rightarrow B$, smooth over $B_{0}=B-\Sigma$ and fixing $(B, \Sigma)$, is a morphism $F:(Y, E) \rightarrow(B, \Sigma) \times T$ with $T=(T, o)$ a germ of a complex manifold, such that $F \mid(B, \Sigma) \times\{o\}=f$. The family $f$ is called rigid if every deformation $F$ of $f$ is induced from $f$ by pullback along the projection $(B, \Sigma) \times T \rightarrow(B, \Sigma)$. Since for a family $Y$ of Abelian varieties the infinitesimal Torelli theorem holds, assuming $f$ is not locally trivial over $B_{0}$, rigidity for $f$ is equivalent to rigidity for the associated period map $p(f): B_{0} \rightarrow \mathcal{A}_{g}$.

Proposition 2.6 ([Pe10, Prop. 3.7.]). Assume that $f$ is not locally trivial over $B_{0}$ or, equivalently, that the period map $p(f)$ is not constant. If the associated Higgs field for $\mathcal{H}_{f}$ is an isomorphism, then $p(f)$ is rigid. In particular, if $f$ reaches the Arakelov bound for $\mathcal{H}_{f}^{1,0}$, the curve $B_{0}$ is rigidly embedded in $\mathcal{A}_{g}$.

\subsection{Application of the slope inequality to fibrations attaining the Arakelov bound}

Theorem 2.7. For a non-isotrivial genus $g \geq 2$ fibration $f: X \rightarrow B$ of semi-stable curves attaining the Arakelov bound ${ }^{16}$, one has $g=2,3,4$.

Proof: Step 1. One first has to make sure that the condition of Theorem 1.5 and Corollary 1.9 are verified, i.e., that $K_{X}+C$ is nef and big for $C=$ $R+\coprod_{s \in \Sigma} X_{s}, R$ the union of certain elliptic tails. If $B$ is not rational, the surface $X$ is minimal: no multisection of $f$ can be rational and $f$ may be assumed to be relatively minimal. Remark 1.6 shows that it suffices to prove that $K_{X}$ is nef, or equivalently [B-P-H-V, VI. Theorem 2.1] that the Kodaira dimension of $X$ is $\geq 0$. Since $g \geq 2$, if $b \geq 1$ the Iitaka-inequality [B-P-H-V, III, Theorem 18.4] shows that in fact $\kappa(X) \geq 1$.

The case $b=0$ remains. Since $f$ is not isotrivial, by [B-P-H-V, III, Theorem 18.2], one has $\operatorname{deg}\left(f_{*} \omega_{X / B}\right)>0$. Since one may also assume that $g \geq 5$, the

\footnotetext{
${ }^{16}$ Recall that one throughout assumes that $2 g(B)-2+|\Sigma|>0$.
} 
Arakelov equality then gives $\operatorname{deg}\left(f_{*} \omega_{X / B}\right)=\frac{1}{2} g(-2+|\Sigma|) \geq 3$. RiemannRoch for $f_{*} \omega_{X}^{\otimes n}$ thus yields the following estimate for the plurigenera

$$
P_{n}(X) \geq H^{0}\left(B, f_{*} \omega_{X / B}^{\otimes n}\right) \geq n \operatorname{deg}\left(f_{*} \omega_{X / B}\right)-2 n+1 \geq n+1
$$

and hence $\kappa(X) \geq 1$. If $X$ is minimal, this finishes the proof. However, $X$ could fail to be minimal. This happens if there exists exceptional curves $E$ which figure as a (multi)-sections.

Write $K_{X}=K_{\min }+\sum E$, where $K_{\min }=\sigma^{*} K_{Y}, \sigma: X \rightarrow Y$ the contraction of all the minimal curves $E$ to a minimal model $Y$. Then $K_{\text {min }}$ is nef on $X$ and so, by the argument of Remark 1.6, $\left(K_{X}+C\right) \cdot D \geq 0$ for all curves $D$ that are not exceptional. Furthermore, since $|\Sigma| \geq 3, E$ being a (multi)section, one has $C \cdot E \geq 3$ and since $K_{X} \cdot E=-1$, it follows that $\left(K_{X}+C\right) \cdot E \geq 2$. Hence $K_{X}+C$ is nef. To show that it is also big, one estimates its self-intersection as follows. Using nefness of $K_{X}+C$ and the previous estimate $\left(K_{X}+C\right) \cdot E \geq 2$, one finds

$$
\begin{aligned}
\left(K_{X}+C\right)^{2} \geq\left(K_{X}+C\right) K_{X}=\left(K_{X}+C\right) \cdot( & \left.K_{\min }+\sum E\right) \\
& \geq 2 \#(\text { exc. curves } E)>0
\end{aligned}
$$

since $X$ is not minimal.

Step 2: Kodaira fibrations cannot attain the Arakelov bound. Indeed, $\omega_{X / B}^{2}=$ $12 \operatorname{deg} f_{*} \omega_{X / B}=12 g(b-1)$ while, by Cor. 1.9

$$
\omega_{X / B}^{2} \leq 4(g-1)(b-1)<12 g(b-1)
$$

It follows that at least one $\delta_{i}(f)$ is non-zero.

Step 3: The case where $\delta_{j}(f)>0$ for some $j>0$. As remarked in Step 1, $\operatorname{deg} f_{*} \omega_{X / B}>0$ since $f$ is not locally trivial and so the Moriwaki slope inequality (16) applies. On the other hand, in the inequality (14), by the assumption on the Arakelov bound, one has $d(f)=\operatorname{deg}\left(f_{*} \omega_{X / B}\right)$, and so 
this inequality combined with Moriwaki's slope inequality gives:

$$
\begin{aligned}
4-\frac{4}{g}+\frac{1}{\operatorname{deg} f_{*} \omega_{X / B}} & {\left[\sum_{s \in \Sigma_{c}} 3 \delta_{s}-\beta(R)-\sum \beta\left(f_{j}\right)\right] } \\
\geq \lambda(f) & =\frac{\omega_{X / B}^{2}}{d(f)} \\
\geq 4 & -\frac{4}{g}+\frac{1}{\operatorname{deg} f_{*} \omega_{X / B}}\left[\sum_{j=1}^{\left[\frac{g}{2}\right]}\left(4 \frac{j(g-j)}{g}-1\right) \cdot \delta_{j}(f)\right] .
\end{aligned}
$$

One gets

$$
\sum_{j=1}^{\left[\frac{g}{2}\right]}\left(\frac{4 j(g-j)}{g}-1\right) \delta_{j}(f)-3 \sum_{s \in \Sigma_{c}} \delta_{s}+\beta(R)+\sum_{j \in J} \beta\left(f_{j}\right) \leq 0 .
$$

Moreover, if the left hand side is zero, then $\beta(X, C)+\sum_{j \in J} \beta\left(f_{j}\right)=0$ where $C=R+\coprod_{s \in \Sigma} X_{s}$.

Rewrite (22) as a sum of terms each involving double points of fixed type. First consider the contribution of double points of type 1. If such a double point belongs to a fiber of non-compact type, it contributes $4\left(1-\frac{1}{g}\right)-1=$ $3-\frac{4}{g}$. On a fiber $X_{s}$ of compact type these are the double points on the semistable elliptic tail $E_{s}+F_{s}$ plus one further point where the tail connects to the remaining components of the fiber, and so there are $\ell_{s}+1$ double points of type 1 in $X_{s}$. Now recall inequality (12): whether or not a connecting rational tail exists or not, the contribution of such an elliptic tail $E_{s}$ to the third and fourth term in $(22)$ is at least $3 \ell_{s}+1$. It follows that the total contribution to $(22)$ is at least

$$
\underbrace{\left(\ell_{s}+1\right)\left(3-\frac{4}{g}\right)}_{\text {1st term }} \underbrace{-3\left(\ell_{s}+1\right)}_{\text {2nd term }}+3 \ell_{s}+1 \geq\left(\ell_{s}+1\right)\left(1-\frac{4}{g}\right) .
$$

So for both situations the "average" contribution from each double point of type 1 is at least $1-\frac{4}{g}$.

For double points of type $j \geq 2$, the first term contributes at least $7-\frac{16}{g}$ and the second term contributes 0 or -3 and so their contribution is at least 
$4-\frac{16}{g}$. Hence, in total, one gets:

$$
\begin{aligned}
0 & \geq \sum_{j=1}^{\left[\frac{g}{2}\right]}\left(\frac{4 j(g-j)}{g}-1\right) \delta_{j}(f)-3 \sum_{s \in \Sigma_{c}} \delta_{s}+\beta(R) \\
& \geq\left(1-\frac{4}{g}\right) \cdot \delta_{1}(f)+\left[4-\frac{16}{g}\right] \cdot \sum_{j \geq 2} \delta_{j}(f)
\end{aligned}
$$

It follows that $g \leq 4$.

Step 4: The case $\delta_{j}(f)=0$ for all $j>0$. I shall argue, following a suggestion of Kang Zuo, that this case does not occur, thereby completing the proof. So suppose it does occur. Note that in that case there are only double points of type 0 . These might include the double points in a string of $(-2)$ curves. However, (22) is an equality and since there are no elliptic tails since $\delta_{1}(f)=0$, the above argument implies that $\beta(X, C)=0$. So, by Theorem 1.5 the open surface $X^{0}=X-C$ does not contain $(-2)$ curves. Furthermore, $X^{0}$ is a locally symmetric space for the group $\operatorname{PSU}(2,1)$ which is of rank 1. So its Baily-Borel compactification $X^{*}$ consists of $X-C$ together with some zero-dimensional boundary components. Since $X^{*}$ is the minimal compactification, there is a holomorphic map $X \rightarrow X^{*}$ which necessarily must contract the curves from $C$, by assumption a non-empty union of semi-stable fibers $X_{s}$, each consisting of an irreducible curve of arithmetic genus $g$ plus, possibly some rational (-2)-curves. As for any fiber configuration, the intersection matrix of the components of $X_{s}$ is negative semi-definite with one dimensional kernel. This is impossible by Mumford's contraction criterion [Mu61] which states that the intersection matrix of a blown down configuration of curves is strictly negative definite. This contradiction shows that this case does not occur.

\section{On the Coleman-Oort conjecture}

\subsection{Special subvarieties of Shimura varieties}

For this subsection the reader is advised to consult [M-O].

To start with, it is helpful to consider bounded symmetric domains $D$ from the perspective of Shimura domains as follows. The group of holomorphic automorphisms of $D$ is the group $G(\mathbf{R})$ of real points of a connected semisimple Q-algebraic group $G$ of adjoint type. It acts transitively on $D$ so that $D=G(\mathbf{R}) / K$ where $K=K_{x}$ is the isotropy group of some point $x \in D$. One 
shows that it comes with a homomorphism

$$
h_{x}: \mathbf{S} \rightarrow G(\mathbf{R}), \quad \mathbf{S}=\operatorname{Res}_{\mathbf{C} / \mathbf{R}} \mathbf{C}^{\times}
$$

where "Res" stands for the Weil restriction; here this simply means that $\mathbf{S}$ is the group $\mathbf{C}^{\times}$considered as a real algebraic group. The group $G$ acts on $h_{x}$ by conjugation and one has a natural identification

$$
D=\left\{\text { conjugacy class of } h_{x}\right\} ; \quad g \cdot x \Longleftrightarrow h_{g \cdot x}=g h_{x} g^{-1} \text {. }
$$

This is the case, because the centralizer of $h_{x}$ is the center of $K_{x}$ so that $h_{g \cdot x}=h_{\tilde{g} \cdot x}$ if and only if $g \tilde{g}^{-1}$ belongs to this center and hence the conjugate by $g$ of $h_{x}$ only depends on the class of $g$ modulo $K_{x}$.

This description of $D$ is now indeed essentially the description of a Shimura domain or a Shimura datum: a pair $(G, D)$ with $G$ a reductive Q-algebraic group and $D$ a conjugacy class of morphisms (23) satisfying some extra technical conditions [Del79, (2.1.1.-3)], which are not important here. For any arithmetic subgroup $\Gamma$ of $G$, the quotient $\Gamma \backslash D$ is called a Shimura variety.

Let $\left(D^{\prime}, G^{\prime}\right)$ be another Shimura domain and let there be given a homomorphism $f: G \rightarrow G^{\prime}$ of $\mathbf{Q}$-algebraic groups. Then $f \circ h_{x}$ defines a point $\bar{f}(x) \in D^{\prime}$ and $(f, \bar{f}):(G, D) \rightarrow\left(G^{\prime}, D^{\prime}\right)$ is an equivariant holomorphic map between bounded symmetric domains. For simplicity, let me assume that $f$ and hence $\bar{f}$ are injective; we thus we may identify $D$ with its image in $D^{\prime}$. One then says that $D$ is a Shimura subdomain of $D^{\prime}$ and if $f(\Gamma) \subset \Gamma^{\prime}$ for some arithmetic subgroups $\Gamma \subset G, \Gamma^{\prime} \subset G^{\prime}$ respectively, one says that $\Gamma \backslash D$ is a special subvariety of the Shimura variety $\Gamma^{\prime} \backslash D^{\prime}$.

\subsection{Special curves in $\mathcal{A}_{g}$}

By Satake's classification [Sat], there are two essentially different classes of Shimura varieties of dimension 1, also called Shimura curves:

- Non-compact Shimura curves. These are precisely the modular curves: quotients of a Shimura domain $(\operatorname{SL}(2), \mathfrak{h})$ by a modular subgroup $\Gamma \subset \mathrm{SL}(2 ; \mathbf{Q})$. After replacing $\Gamma$ by a suitable finite index subgroup, these carry a universal family $E_{\Gamma} \rightarrow C_{\Gamma}=\Gamma \backslash \mathfrak{h}$ of elliptic curves. Here $\mathfrak{h}$ denotes the upper half plane.

- Compact Shimura curves which all arise as follows. Let $F$ be a totally real number field and $\mathbf{D}$ a quaternion algebra over $F$ which splits at 
exactly one place. Consider

$$
\mathrm{SU}(1, \mathbf{D})=\left\{x \in \mathbf{D}^{\times} \mid \operatorname{Nrd}_{\mathbf{D}}(x)=1\right\}
$$

and the arithmetic subgroup

$$
\Gamma_{\mathbf{D}}=\left\{x \in \text { some fixed maximal order of } \mathbf{D} \mid \operatorname{Nrd}_{\mathbf{D}}(x)=1\right\}
$$

Then, $\left(\operatorname{Res}_{F / \mathbf{Q}} \mathrm{SU}(1, \mathbf{D}), \mathfrak{h}\right)$ is a Shimura datum ${ }^{17}$ and

$$
C_{\Gamma_{\mathbf{D}}}:=\Gamma_{\mathbf{D}} \backslash \mathfrak{h}
$$

is a compact Shimura curve. Note that with $\mathbf{H}$ the algebra of the quaternions, one has

$$
\begin{aligned}
& \mathbf{D}(\mathbf{R}) \simeq M(2 ; \mathbf{R}) \otimes_{\mathbf{R}} \underbrace{\mathbf{H} \otimes_{\mathbf{R}} \cdots \otimes_{\mathbf{R}} \mathbf{H}}_{m-1}, \quad m=[F: \mathbf{Q}] \\
& G(\mathbf{R}) \simeq \mathrm{SL}(2, \mathbf{R}) \times \underbrace{\mathrm{SU}(2) \times \cdots \times \mathrm{SU}(2)}_{m-1} .
\end{aligned}
$$

which shows that indeed, the upper half plane is the associated bounded domain. Again, after possibly replacing $\Gamma_{\mathbf{D}}$ by a subgroup of finite index, there is a universal family of abelian varieties $A_{\Gamma_{\mathbf{D}}} \rightarrow C_{\Gamma_{\mathbf{D}}}$ over this Shimura curve. The fibers of this family turn out to have dimension $g=2^{m-1}$ or $g=2^{m}$.

In order to classify special curves in $\mathcal{A}_{g}$, one needs to understand the embeddings of these curves in $\mathcal{A}_{g}$ as special subvarieties. There are many non-rigidly embedded Shimura curves; this depends on the arithmetic of the corresponding embedding of $\mathbf{Q}$-algebraic groups $G \rightarrow \operatorname{Sp}(g)$, where $G$ is the Q-simple group of the type introduced above (i.e., $G(\mathbf{R})$ has precisely one non-compact factor, $\operatorname{SL}(2 ; \mathbf{R}))$. In view of Prop. 2.6 , I shall only consider rigidly embedded Shimura curves. The result is as follows:

Proposition 3.1. Each of the two types of Shimura curves $C_{\Gamma}$, respectively $C_{\Gamma_{D}}$ can be rigidly embedded in $\mathcal{A}_{g}$ for some $g$. For $k$ large enough, one also gets a rigid embedding of $C_{\Gamma}$, respectively $C_{\Gamma_{D}}$ in the fine moduli space $\mathcal{A}_{g, k}$ of polarized Abelian varieties of dimension $g$ with level $k$-structure.

\footnotetext{
${ }^{17}$ As before "Res" stands for the Weil restriction; here it means that the $F$-group in question is to be considered as a group over the field $\mathbf{Q}$.
} 
1. For the first type, $g$ can be arbitrary and the modular family of Abelian varieties is isogeneous to a fibered product

$$
\underbrace{E_{\Gamma} \times{ }_{C_{\Gamma}} \cdots \times{ }_{C_{\Gamma}} E_{\Gamma}}_{g \text { copies. }} .
$$

2. For the second type, $g$ is a multiple of $g=2^{m}$ or $2^{m-1}, m=[F: \mathbf{Q}]$ as before and the modular family is isogenous to a fibered product

$$
A_{\Gamma_{D}} \times C_{\Gamma_{D}} \cdots \times{ }_{C_{\Gamma_{D}}} A_{\Gamma_{D}}
$$

of the modular family over a Mumford curve.

Sketch of proof. If follows from [Sai93, §6] that rigid representations in the sense of $[\mathrm{Ad}]$ give precisely the rigid embeddings we are classifying.

In loc. cit. it is explained that symplectic representations are all constructed as follows. As before, let $F$ be a totally real number field and $\mathbf{D}$ a quaternion algebra over $F$ which splits at exactly one place. For simplicity, assume that $F / \mathbf{Q}$ is Galois with Galois group $\mathcal{G}$. The set of real embeddings $i_{\alpha}: F \hookrightarrow \mathbf{R}, \alpha=1, \ldots, m$ is identified with $S=\{1, \ldots, m\}$, the unique noncompact place being the first. We have

$$
G^{\alpha}=\mathrm{SU}(1, \mathbf{D}) \otimes_{i_{\alpha}} \mathbf{R}, \quad G^{\alpha}(\mathbf{C})=\mathrm{SL}\left(V^{\alpha}, \mathbf{C}\right), \quad V^{\alpha}=\mathbf{C}^{2} .
$$

The representations $V^{\alpha}$ are called "atoms"; "molecules" are the tensor representation $V^{\alpha_{1}} \otimes \cdots \otimes V^{\alpha_{s}}, \alpha_{1}, \ldots, \alpha_{s} \in S$ and a direct sum of tensor representations is called a "polymer". The Galois group $\mathcal{G}$ acts on polymers. Polymers giving rigid representations are $\mathcal{G}$-stable polymers consisting of a special type of molecules: If $m=1$ only one atom per molecule is allowed and if $m>1$ each molecule contains exactly one "non-compact" atom, i.e., $V^{1}$. Note that if $m=1$ the only such polymers are direct sums of the atom $V^{1}$ and in the second case one can only have direct sums of the molecule $V^{1} \otimes \cdots \otimes V^{m}$. If $m=1$ these representations are defined over $\mathbf{Q}$ and below a symplectic embedding is exhibited. For $m>1$ some more work is need.

Case 1. Non-compact embedded curves. Satake in [Sat] classifies embeddings of algebraic groups $G \hookrightarrow \operatorname{Sp}(g)$ for $G$ a $\mathbf{Q}$-simple algebraic group and $G(\mathbf{R})$ without compact factors. For $G=\mathrm{SL}(2)$ such a Satake embedding is induced by the following symplectic embedding. Set $V_{k}=\left(\mathbf{R}^{2 k}, J_{k}\right)$, $J_{k}=\left(\begin{array}{cc}0 & \mathbf{1}_{k} \\ -\mathbf{1}_{k} & 0\end{array}\right)$. The direct sum $\oplus_{k} V_{1}$ is isomorphic to the symplectic 
space $V_{k}$. Whence a faithful representation $\rho_{k}$ of $\mathrm{SL}(2)$ :

$$
\left(\begin{array}{ll}
a & b \\
c & d
\end{array}\right) \stackrel{\rho_{k}}{\longrightarrow}\left(\begin{array}{ll}
a \mathbf{1}_{k} & b \mathbf{1}_{k} \\
c \mathbf{1}_{k} & d \mathbf{1}_{k}
\end{array}\right)
$$

For any $k=1, \ldots, g$ the direct sum representation $\rho_{k} \oplus$ (rank $(g-k)$ trivial representation) induces a holomorphic embedding $\mathfrak{h} \hookrightarrow \mathfrak{h}_{g}$. It gives the non-compact embedded Shimura curves starting from the Shimura datum $(\mathrm{SL}(2), \mathfrak{h})$. There is no locally constant factor if and only if $k=g$ and then the embedding is rigid. The non-compact rigid curves are often called rigid curves of Satake type. From its description one sees that the universal family is the fibered self product of the universal family of elliptic curves over the upper half plane.

Case 2. Symplectic embeddings when compact factors are present. Let $\mathbf{D}^{\alpha}$ be the twisted $F$-algebra $D$ obtained via the embedding $i_{\alpha}$ and form the "corestriction from $F$ to $\mathbf{Q}$ of the algebra D":

$$
\mathbf{E}=\operatorname{Cor}_{F / \mathbf{Q}} \mathbf{D}:=\mathbf{D}^{1} \otimes_{F} \cdots \otimes_{F} \mathbf{D}^{m}
$$

There are two possibilities for this algebra:

$$
\mathbf{E}= \begin{cases}M\left(2^{m}, \mathbf{Q}\right) & \text { (split case) } \\ M\left(2^{m-1}, H\right), H \text { a quaternion algebra } / \mathbf{Q} & \text { (non-split case) }\end{cases}
$$

The $G$-representation space

$$
W=V_{1} \otimes V_{2} \otimes \cdots \otimes V_{m}, \quad V_{\alpha}=\mathbf{C}^{2} \text { with } G^{\alpha}(\mathbf{C}) \text { acting }
$$

turns out to be a symplectic representation over $\mathbf{Q}$ in the split case ; in the non-split case this is true for $W \oplus W$. See [Ad, Sect. 4] or [Vie-Z, Section 5]. The corresponding embedding

$$
\mathfrak{h}_{1} \hookrightarrow \mathfrak{h}_{g}, \quad g=2^{m-1}, \text { resp. } g=2^{m}
$$

is then a rigid embedding, as explained above. It is called an embedded curve of Mumford type. All other embeddings come from direct sums of such representations.

Remark. A different proof of this classification can be found in [Vie-Z]. 


\subsection{Relation with the Arakelov bounds}

Let me summarize the pertinent results so far. First of all, the base curve of any family of $g$-dimensional polarized Abelian varieties over a curve attaining the Arakelov bound must be a Shimura curve embedded in $\mathcal{A}_{g}$ as a rigid curve of Satake type or of Mumford type. If such a curve is also the base curve of a family of curves of genus $g$, then $g \leq 4$.

Conversely, an embedded Shimura curve $C_{0} \subset \mathcal{A}_{g}$ of the above type and which is generically contained in Torelli locus need not carry a family of semistable curves over it, since $\mathcal{A}_{g}$ is not a fine moduli space. Passing to a suitable fine moduli space $\mathcal{A}_{g, m}$ destroys the injectivity of the period map: it becomes a branched double cover which is precisely branched in the intersection of the locus of the hyperelliptic curves and the Torelli locus. For curves not meeting this locus, it of course still follows that $g \leq 4$. However, one cannot a priori exclude special curves $C_{0} \subset \mathcal{A}_{g, m}$ that meet the hyperelliptic locus.

Let me give a brief outline the proof of Theorem (B) as stated in the Introduction. Let $C$ be the Zariski closure of $C_{0}$ in the moduli space $\overline{\mathcal{M}}_{g, m}$ of semi-stable curves and set

$$
\mathcal{H}_{g}=\text { the closure of the hyperelliptic locus in } \overline{\mathcal{M}}_{g . m}
$$

Now either $C \subset \mathcal{H}_{g}$ or $C$ meets the hyperelliptic locus in a finite set of points.

For the first case, see [L-Z]. In the second case, form the double cover $\pi: B \rightarrow C$ branched in the points of $\mathcal{H}_{g} \cap C$. This produces a surface $f$ : $X \rightarrow B$ fibered in genus $g$ curves. A little care is needed here: the fiber over a point in the ramification locus might be singular, but at most of compact type. As we have seen, this is because the closure of the Torelli locus in $\mathcal{A}_{g . m}$ might contain singular curves of compact type.

On $B$ the Arakelov bound is then no longer valid: by [L-Z2, Proposition $1.9]$, one has to replace it by

$$
\begin{gathered}
d(f)=\operatorname{deg} \mathcal{H}_{f}^{1,0}=\operatorname{deg} f_{*} \omega_{S / B}=\frac{1}{2} \cdot g \cdot\left(\operatorname{deg} \Omega_{B}^{1}(\log (\Sigma))-h_{0}\right), \\
h_{0}=\left|\mathcal{H}_{g} \cap C_{0}\right|
\end{gathered}
$$


This follows directly from the Hurwitz formula for the double cover $\pi$. One can use this to rewrite (14) as

$$
\begin{array}{r}
4\left(1-\frac{1}{g}\right)+\frac{1}{d(f)} \cdot\left(2(g-1) h_{0}+\sum_{s \in \Sigma_{c}} 3 \delta_{s}-\beta(R)-\sum_{j \in J} \beta\left(f_{j}\right)\right) \\
\geq \lambda(f)=\frac{\omega_{X / B}^{2}}{d(f)}
\end{array}
$$

However, one needs a better inequality. To state it conveniently, some further notation is used:

$$
\begin{aligned}
n_{j, s} & =\# \text { of components of genus } j \text { in } X_{s}, \\
\epsilon_{s} & =\left(\sum_{j \geq 1} n_{j, s}\right)-1 \\
\theta_{s} & =\left(\sum_{j \geq 2} 3 n_{j, s}+2 n_{1, s}\right)-3 \\
\Lambda_{0} & =\mathcal{H}_{g} \cap C_{0} .
\end{aligned}
$$

Then by [L-Z2, Theorem 1.13] one has:

$$
\begin{gathered}
4\left(1-\frac{1}{g}\right)+\frac{2(g-1) h_{0}}{d(f)}+\frac{1}{d(f)} \cdot\left(\frac{3}{2} \sum_{s \in \Sigma_{c} \cap \Lambda_{0}} \epsilon_{s}+\sum_{s \in \Sigma_{c}-\Lambda_{0}} \theta_{s}\right) \\
\geq \lambda(f)=\frac{\omega_{X / B}^{2}}{d(f)} .
\end{gathered}
$$

This inequality turns out to be a consequence of the refined BMY-inequality by pulling back the family along a carefully chosen ramified cover of the base curve $B$ and replacing $R$ by another curve.

In order to estimate $h_{0}$, the idea is to replace the Moriwaki inequality by a different slope inequality in which $h_{0}$ also appears. This refined slope inequality from [L-Z2, Theorem 1.14] is a highly non-trivial result which depends on an analysis of a morphism between auxiliary Higgs bundles and a fine analysis of the singularities of the hyperelliptic singular fibers. It is valid as soon as $g \geq 7$ and reads as follows:

$$
\lambda(f) \geq \frac{5 g-6}{g}+\frac{1}{d(f)} \cdot\left[2(g-2) h_{0}\right]+\frac{1}{d(f)} \cdot\left[\sum_{s \in \Sigma_{c} \cap \Lambda_{0}} 2 \epsilon_{s}+\sum_{s \in \Sigma_{c}-\Lambda_{0}} \theta_{s}\right]
$$


Comparing with (24), one gets a bound for $h_{0}$ :

$$
\frac{2 h_{0}}{d(f)} \geq \frac{g-2}{g}+\frac{1}{d(f)} \cdot\left[\sum_{s \in \Sigma_{c} \cap \Lambda_{0}} \frac{1}{2} \epsilon_{s}\right] \geq \frac{g-2}{g} .
$$

Here one uses that $\epsilon_{s}=\left(\sum_{j \geq 1} n_{j, s}\right)-1 \geq 0$ since there must be non-rational components in the fiber $X_{s}$.

Now use this bound together with the primitive upperbound (5), together with (25) as follows:

$$
12 \geq \lambda(f) \geq \frac{5 g-6}{g}+\frac{(g-2)^{2}}{g}+\text { remaining term. }
$$

where the remaining term is the non-negative expression

$$
\frac{1}{d(f)} \cdot\left[\sum_{s \in \Sigma_{c} \cap \Lambda_{0}} 2 \epsilon_{s}+\sum_{s \in \Sigma_{c}-\Lambda_{0}} \theta_{s}\right]
$$

It follows that

$$
0 \geq \frac{5 g-6}{g}+\frac{(g-2)^{2}}{g}-12=\frac{g^{2}-11 g-2}{g} \Longrightarrow g \leq 11 .
$$

Remarks 3.2. 1) Non-rigidly embedded curves also have been classified; in fact in [Ad, Sai93] one finds a classification scheme for all special subvarieties of $\mathcal{A}_{g}$. The variation of Hodge structures corresponding to non-rigid curve embeddings might be decomposable over $\mathbf{Q}$ and the corresponding Higgs bundles may contain unitary subsystems. The number of possibilities depend on $g$ and the field $F$ over which the quaternion algebra is defined. As $g$ grows and the index $[F: \mathbf{Q}]$ grows, the number of possibilities grows astronomically.

2) Let $C_{0}=C-\Delta$ be a curve contained in the Torelli locus. By the original Arakelov result, any such curve is rigidly embedded in $\mathcal{M}_{g}$ and there are only finitely many of these due to the boundedness statement in Arakelov's theorem. So, fixing $(C, \Delta)$ with $C_{0}$ a Shimura curve, there are only finitely many embeddings of $C_{0}$ whose image is generically contained in the Torelli locus. In particular, among the infinitely many possible embeddings $C_{0} \hookrightarrow \mathcal{A}_{g}$, only finitely many can be generically contained in the Torelli locus. In particular, if $[F: \mathbf{Q}]$ is large, such a curve is not contained in it.

3) Jacobians of smooth curves have an irreducible polarization which give Q-irreducible variations of Hodge structures. However, the corresponding 
Higgs bundles (which only "see" the R-structure) can decompose and hence could in particular contain unitary Higgs subbundles defined over some real number field $\neq \mathbf{Q}$. Note that for such a subbundle the monodromy need no longer be finite. Indeed, this can be seen to happen for the examples in [M-O]. See also [Cat-De].

\section{References}

[Ad] Addington, S.: Equivariant holomorphic maps of symmetric domains, Duke Math. Journal 55 (1987), 65-88.

[Ar] Arakelov, A.: Families of algebraic curves with fixed degeneracies, Izv. Akad. Nauk. SSSR, Ser. Math. 35 ( 1971), 1277-1302.

[A-C-G] Arbarello, E., M. Cornalba and Ph. Griffiths: The geometry of algebraic curves, II Grundlehren de Math. Wissensch. 268, Springer Verlag (2010).

[B-P-H-V] Barth, W., C. Peters, K. Hulek And A. van de Ven: Compact Complex Surfaces (second enlarged edition), Springer Verlag (2004).

[Fal] Faltings, G.: Arakelov's theorem for abelian varieties, Inv. Math. 73 (1983), 337-348.

[Ca-MS-P] Carlson, J., S. Müller-Stach and C. Peters: Period Mappings and Period Domains, Cambridge Studies in advanced math. 85, Cambridge Univ. Press, Cambridge (2003).

[Cat-De] Catanese, F. And M. Dettweiler: Vector bundles on curves coming from Variation of Hodge Structures, arXiv:1505.05064 [math. AG].

[Ch-Y] Cheng, S. Y. AND S.-T. YAU: Inequality between Chern numbers of singular Kähler surfaces and characterizations of orbit space of discrete group of $S U(2,1)$, Contemporary Math., 49, World Sci. Publishing, Singapore, (1986), 31-43.

[C-L-Z2] Chen, K., X. Lu And K. ZuO: On the Oort conjecture for Shimura varieties of unitary and orthogonal types, http://arxiv.org/abs/1410.5739.

[Co] Coleman, R.: Torsion points on curves, in Galois representations and arithmetic algebraic geometry (Kyoto 1985/Tokyo 1986), 235-247, Adv.

Math. Stud. Pure Math. 12 North-Holland, Amsterdam 1987. 
[Del79] Deligne, P.: Variétés de Shimura: interprétation modulaire, et techniques de construction de modèles canoniques in Automorphic forms, representations and L-functions (Proc. Sympos. Pure Math., Oregon State Univ., Corvallis, Ore., 1977), Part 2 247-289, Proc. Sympos. Pure Math., XXXIII, Amer. Math. Soc., Providence, R.I., (1979).

[Jost-Zuo] Jost, J., AND K. ZuO: Arakelov type inequalities for Hodge bundles over algebraic varieties, Part I: Hodge bundles over algebraic curves with unipotent monodromies around singularities, J. Alg. Geom. 11 (2002), 535-546.

[Ko] Kobayashi, R.: Einstein-Kaehler metrics on open algebraic surfaces of general type, Tôhoku Math. J. 37 (1985), 43-77.

[L-T-Y-Z] LU, J., S-Li TAN, F. YU AND K. ZuO: A new inequality on the Hodge number $h^{1,1}$ of algebraic surfaces, http://arxiv.org/abs/1303.2749.

[L-Z] LU, X. And K. ZuO: On Shimura curves in the Torelli locus of curves, http://arxiv.org/abs/1311.5858.

[L-Z2] Lu, X. AND K. ZuO: The Oort conjecture on Shimura curves in the Torelli locus of curves, http://arxiv.org/abs/1405.4751

[L-Z3] Lu, X. AND K. ZuO: On the slope of hyperelliptic fibrations with positive relative irregularity, http://arxiv.org/abs/1311.727.

[L-Z4] Lu, X. And K. Zuo: On the slope conjecture of Barja and Stoppino for fibred surfaces, http://arxiv.org/abs/1504.06276.

[L-Z5] Lu, X. AND K. ZuO: On the Severi type inequalities for irregular surfaces, http://arxiv.org/abs/1504.06569.

[Miy] MryaokA, M.: The maximal number of quotient singularities on surfaces with given numerical invariants, Math. Ann. 268 (1984), 159-171.

[M-O] Moonen, B. And F. OorT: The Torelli locus and special subvarieties, Handbook of moduli. Vol. II, 549-594, Adv. Lect. Math. (ALM), 25, Int. Press, Somerville, MA, 2013.

[Mor] MorIWAKI, : Relative Bogomolov's inequality and the cone of positive divisors on the moduli space of stable curves, J. Am. Math. Soc. 11 (1989), 569-600.

[Mu61] Mumford, D.: Topology of normal singularities and a criterion for simplicity, Publ. Math. IHES 36 (1961), 229-246. 
[Mum69] Mumford, D.: A note on Shimura's paper "Discontinuous groups and abelian varieties", Math. Annalen 181 (1969), 345-351.

[Mum75] Mumford, D.: Curves and Their Jacobians, Univ. of Michigan Press, Ann Arbor (1975).

[O] Oort, F.: Canonical liftings and dense sets of CM-points, in: Arithmetic geometry (Cortona, 1994), 228-234, Sympos. Math., XXXVII, Cambridge Univ. Press, Cambridge, (1997).

[Pe00] Peters, C.: Arakelov inequalities for Hodge bundles, Prépub. de l'Inst. Fourier 511 (2000).

[Pe10] Peters, C.: Rigidity, Past and Present, Proceedings of Teichmueller Theory and Moduli Problems (held at HRI, India in January 2006), Ramanujan Mathematical Society's Lecture Notes Series 10 (2010), $529-548$.

[PeSt] Peters, C. And J. Steenbrink: Mixed Hodge Structures, Springer Verlag, Berlin etc. (2008).

[Sai93] SAIto, MA.: Classification of non-rigid families of abelian varieties, Tôhoku Math. J. 45 (1993), 159-189.

[Sak] SAKAI, F.: Semistable curves on algebraic surfaces and logarithmic pluricanonical maps, Math. Ann. 254 (1980), 89-120.

[Sat] SATAKE, I.: Symplectic representations of algebraic groups satisfying a certain analyticity condition, Acta Math. 117 (1967), 215-279.

[Vie-Z] VIEHWEG, E AND K. ZuO: A characterization of certain Shimura curves in the moduli stack of abelian varieties, J. Differential Geom. 66 (2004), 233-287.

[Xiao] XIAo, G.: Fibered algebraic surfaces with low slope, Math. Ann. 276 (1987), 125-148.

Chris Peters

Institut Fourier, Université de Grenoble Alpes

\& Technical University Eindhoven

E-mail: c.a.m.peters@tue.nl 